\title{
Elastic constants from microscopic strain fluctuations
}

\author{
Surajit Sengupta ${ }^{1 *}$, Peter Nielaba ${ }^{2},{\text { Madan } \text { Rao }^{3 \dagger} \text { and K. Binder }}^{1}$ \\ ${ }^{1}$ Institut für Physik, Johannes Gutenberg Universität Mainz, 55099, Mainz, Germany \\ 2 Universität Konstanz, Fakultät für Physik, Fach M 691, 78457, Konstanz, Germany \\ ${ }^{3}$ Raman Research Institute, C. V. Raman Avenue, Bangalore 560080, India
}

(June 22, 2021)

\begin{abstract}
Fluctuations of the instantaneous local Lagrangian strain $\epsilon_{i j}(\mathbf{r}, \mathbf{t})$, measured with respect to a static "reference" lattice, are used to obtain accurate estimates of the elastic constants of model solids from atomistic computer simulations. The measured strains are systematically coarse - grained by averaging them within subsystems (of size $L_{b}$ ) of a system (of total size $L$ ) in the canonical ensemble. Using a simple finite size scaling theory we predict the behaviour of the fluctuations $\left\langle\epsilon_{i j} \epsilon_{k l}>\right.$ as a function of $L_{b} / L$ and extract elastic constants of the system in the thermodynamic limit at nonzero temperature. Our method is simple to implement, efficient and general enough to be able to handle a wide class of model systems including those with singular potentials without any essential modification. We illustrate the technique by computing isothermal elastic constants of "hard" and "soft" disk triangular solids in two dimensions from Monte Carlo and molecular dynamics simula-
\end{abstract}

\footnotetext{
*on leave from: Material Science Division, Indira Gandhi Centre for Atomic Research, Kalpakkam 603102, India

$\dagger$ on leave from: Institute of Mathematical Sciences, Taramani, Chennai 600113, India
} 
tions. We compare our results with those from earlier simulations and theory.

Typeset using REVTEX 


\section{INTRODUCTION}

One is often interested in long length scale and long time scale phenomena in solids ( eg. late stage kinetics of solid state phase transformations [1,2]; motion of domain walls interfaces [3]; fracture [5]; friction [4] etc.). Such phenomena are usually described by continuum theories. Microscopic simulations [6] of finite systems, on the other hand, like molecular dynamics, lattice Boltzmann or Monte Carlo, deal with microscopic variables like the positions and velocities of constituent particles and together with detailed knowledge of interatomic potentials, hope to build up a description of the macro system from a knowledge of these micro variables. How does one recover continuum physics from simulating the dynamics of $N$ particles? This requires a "coarse-graining" procedure in space (for equilibrium) or both space and time for non-equilibrium continuum theories. Over what coarse graining length and time scale does one recover results consistent with continuum theories? In this paper, we attempt to answer these questions for the simplest nontrivial case, namely, a crystalline solid, (without any point, line or surface defects [8]) in equilibrium, at a non zero temperature far away from phase transitions. We show that coarse graining of microscopic local strain fluctuations obtained from configurations generated in a computer simulation, enables us to calculate elastic susceptibilities (compliances) as a function of the coarse graining length. Detailed finite size scaling analysis of this data yields finally elastic constants of the solid the essential inputs to continuum elasticity theory [7]. The strain field (together with defect densities) constitute the coarse grained description of a solid essential for understanding solid state phase transitions like structural transitions [10,9] and melting [11 13].

Calculation of elastic constants from simulations fall into two categories, viz. they are obtained either from, thermal averages of fluctuations of the stress or the strain - the so called "fluctuation" methods [14], or from the stress - strain curve as computed from a series

of simulations [15]. Fluctuation methods, though requiring longer runs for accumulating statistically significant data, are often preferred because the entire matrix of elastic constants can be evaluated in a single run, whereas in the latter method for every elastic constant an 
appropriate strain (or stress) have to be applied. Also mapping out the stress - strain curve can be treacherous especially for soft systems where the possibility of setting up a plastic flow in the system is high [37]. Though for most systems careful applications of either procedure should yield results of comparable accuracy, they suffer from some common limitations. Firstly, elastic constants are obtained for a particular system size $L$. In order to take the infinite size (thermodynamic) limit one needs to simulate a sequence of systems with increasing values of $L$ - often a computationally expensive proposition since equilibration of large systems take increasingly larger times. Secondly, these procedures are not general and fail for model systems of particles interacting via singular potentials eg., the hard sphere [16,17] or the hard disk [18] system where the instantaneous force on a particle is not well defined. To obtain elastic constants of hard systems, one therefore needs to develop special methods [16,17]. For instance, the elastic constants of the hard sphere system was calculated by Runge and Chester [17] by generalizing a technique used previously for calculating the hard sphere pressure [19]. In this approach one tries to evaluate ensemble averages of quantities involving delta functions by calculating acceptance probabilities of virtual Monte Carlo steps. Such methods are cumbersome to use and may require ill defined averages of quantities whose variance has weak divergences [17]. In contrast, the method presented here has several advantages. Firstly, elastic constants are obtained from a coarse graining procedure which automatically, gives the infinite system values. Secondly, our procedure needs only the instantaneous particle configurations and makes no reference to the potential or forces. It is therefore quite generally applicable to any system for which these configurations or "snapshots" are known. Lastly, it will soon be evident that this technique is easy to use requiring a computational effort not much more than a calculation of, say, the pair distribution function [6] for a given particle configuration.

There are three essential elements or steps in the method,

1. a procedure for calculating elastic strains from configurations.

2. the coarse graining procedure of averaging fluctuations of these strains over larger and 
larger sub -blocks of length $L_{b} \leq L$ of a system of total size $L$ and calculating the values of these fluctuations in the thermodynamic limit

3. and, finally, converting the data for the strain fluctuations into elastic constants using well known results of continuum elasticity theory [7,20].

We take up the second of these steps first as it is quite general and applicable to fluctuations of any intensive variable. Indeed, variants of this method have been used extensively 21 24,27 in the past for obtaining finite size scaled susceptibilities and goes by the name of "block - analysis". In the next section (section II) we describe this block -analysis technique for obtaining the susceptibility of the two -dimensional Ising model [24] for illustration. In section III we define the microscopic strains $\epsilon_{i j}(\mathbf{r})$ and describe how we obtain them from our computer simulations. We then explain how to adopt the coarse graining scheme described in section II for strains to finally obtain the elastic constants. This is followed by our results for the hard disk and the inverse twelveth power "soft" disk systems in two dimensions. We conclude this paper with a discussion of these results and enumerating future directions.

\section{FINITE SIZE SCALING OF FLUCTUATIONS IN SUBSYSTEMS}

The block analysis method has been used to obtain the compressibility of the Ising lattice gas [22], the two -dimensional Lennard -Jones fluid [23,24] and fluids with internal classical and quantum degrees of freedom [28,29]. Below, we describe a version of this method which allows us to explicitly and systematically incorporate finite size effects arising both from a non -zero correlation length as well as special constraints due to the nature of the ensemble used. This treatment is analogous to the one used in Ref. [27] for analyzing density fluctuations in the two -dimensional Lennard Jones fluid.

To begin, consider a general system described by a scalar order parameter $\phi(\mathbf{r})$. We are interested in obtaining thermodynamic properties of this system in the disordered phase. It is therefore sufficient to use the following quadratic Helmholtz free energy functional, $F[\{\phi\}]$. 


$$
F=k_{B} T \int d^{d} r\left(\frac{1}{2} r \phi^{2}+\frac{1}{2} c\{\nabla \phi(\mathbf{r})\}^{2}\right)
$$

The correlation function $G_{\phi \phi}(q)$ implied by the above free energy in the high temperature phase $(\langle\phi\rangle=0)$ is given [8] by the following Ornstein -Zernicke form,

$$
\beta G_{\phi \phi}(q)=<\phi_{q} \phi_{-q}>=\mathcal{X}_{\phi \phi}^{\infty} \frac{1}{1+\left(q \xi_{\phi \phi}\right)^{2}}
$$

Where $\xi_{\phi \phi}$ is the correlation length $(=\sqrt{c / r})$, the (infinite system) susceptibility $\mathcal{X}_{\phi \phi}^{\infty}=<$ $\phi^{2}>$ is, in turn, given by $\lim _{q \rightarrow 0} \beta G_{\phi \phi}(q), \beta=\left(k_{B} T\right)^{-1}$ and the angular brackets $<\ldots>=$ $\int[d \phi] \exp (-\beta F[\{\phi\}])$.

Consider further that we want to measure this susceptibility from a computer simulation of an Ising model within a finite simulation box of size $L$. In the course of the simulation we measure $\phi$ averaged within a sub-block of size $L_{b} \leq L$,

$$
\bar{\phi}=L_{b}^{-d} \int^{L_{b}} d^{d} r \phi(\mathbf{r})
$$

Then the fluctuations of $\bar{\phi}$ measured within this block is,

$$
\begin{aligned}
<\bar{\phi}^{2}>_{L_{b}} L_{b}{ }^{d} & ={L_{b}}^{-d} \int^{L_{b}} d^{d} r^{\prime} d^{d} r<\phi(r) \phi\left(r^{\prime}\right)> \\
& =\int^{L_{b}} d^{d} r \beta G_{\phi \phi}(r), \\
& \equiv \mathcal{X}_{\phi \phi}^{L_{b}}
\end{aligned}
$$

Where $G_{\phi \phi}(r)$ is the inverse Fourier transform of the correlation function defined in Eq.(2) and is given [8], quite generally, by,

$$
G_{\phi \phi}(r)=\xi^{-2} \mathcal{X}_{\phi \phi}^{\infty}|r|^{(d-2)} Y\left(|r| / \xi_{\phi \phi}\right)
$$

where,

$$
Y(\eta)=\int_{0}^{\infty} z^{d-1} d z \int(2 \pi)^{-d} d \Omega_{d} \frac{e^{i z \cos \theta}}{\left[z^{2}+\eta^{2}\right]}
$$

One expects therefore that as, $L_{b} \rightarrow L$ the block susceptibility $\mathcal{X}_{\phi \phi}^{L_{b}} \rightarrow \mathcal{X}_{\phi \phi}^{\infty}$. The behaviour of the block susceptibilities, however, is strongly dependent on the ensemble [30] in 
which the simulation is carried out. For example, using the lattice gas [8] language, in a "grand canonical" ensemble where the chemical composition $\phi$ of the lattice gas is allowed to fluctuate keeping the chemical potential difference fixed ( $=0$ in this case) the block susceptibility does approach $\mathcal{X}_{\phi \phi}^{\infty}$ for large $L_{b}$. In a canonical ensemble, however, the average of $\phi$ over the entire system is constrained to vanish for all times. In such a case the behaviour of $\mathcal{X}_{\phi \phi}^{L_{b}}$ is more complicated but can, nevertheless, be explicitly determined as follows.

Introducing the Lagrange multiplier $\kappa$ and defining the new correlator,

$$
G^{\prime}(r)=\int[d \phi] \phi(0) \phi(r) \exp \left(-\beta F-\kappa \int d^{d} r \phi\right)
$$

where the free energy $F$ is given by Eq.(11)), one shows that that $G_{\phi \phi}^{\prime}(r)=G_{\phi \phi}(r)-\Delta_{L}$. The constant $\Delta_{L}$ is given by,

$$
\Delta_{L}=\frac{1}{L^{d}} \int^{L} d^{d} r G_{\phi \phi}(r)
$$

Repeating the analysis with $G_{\phi \phi}(r)$ replaced by $G_{\phi \phi}^{\prime}(r)$ one obtains the desired finite size scaling form for $\mathcal{X}_{\phi \phi}^{L_{b}}$ in the canonical ensemble. We derive this form explicitly for the case of $d=2$ below.

The correlation function $G(r)$ is,

$$
G(r)=\frac{2}{\pi} \xi^{-2} \mathcal{X}^{\infty} K_{0}(r / \xi)
$$

where $K_{0}$ is a Bessel function and we have suppressed the subscripts in $G, \mathcal{X}$ and $\xi$ for simplicity. We have therefore,

$$
\Delta_{L}=\mathcal{X}^{\infty} L^{-2} \Psi(L / \xi)
$$

with the function $\Psi(\alpha)$ being defined as,

$$
\Psi(\alpha)=\frac{2}{\pi} \alpha^{2} \int_{0}^{1} \int_{0}^{1} d x d y K_{0}\left(\alpha \sqrt{x^{2}+y^{2}}\right)
$$

As can be easily verified $\Psi(\alpha)$ goes to 1 within a range $\mathcal{O}(1)$. Using the above expressions we find finally, 


$$
\mathcal{X}^{L_{b}}=\mathcal{X}^{\infty}\left[\Psi(x L / \xi)-\Psi(L / \xi) x^{2}\right] .
$$

This is the main result of this section.

Note that the form given above implies $\mathcal{X}^{L_{b}} \rightarrow 0$ as $L_{b} \rightarrow L$. In general there will be higher order corrections to Eq.(11) coming from higher order correlations signifying the breakdown of the quadratic form for the free energy Eq.(11). These introduce terms of order $x^{(2 N)}$ implying extra parameters (with the constraint $\mathcal{X}^{L}=0$ being imposed to eliminate one of them). In Fig. (1) we have plotted $\mathcal{X}^{L_{b}} \times x$ against $x$ for various values of $L / \xi$. One observes that for $L / \xi \gg 1, \Psi(\alpha) \rightarrow 1$ and Eq.(11) goes over to the following simple limiting form,

$$
\mathcal{X}^{L_{b}} \times x=\mathcal{X}^{\infty}\left[x-x^{3}\right] .
$$

One can then extract $\mathcal{X}^{\infty}$ from the slope of the linear region at small $x$. This is equivalent to the procedure followed for example in Ref. [24] for extracting the compressibility of the lattice gas at the critical density. Obviously, for $L / \xi \sim 1$, this construction becomes less well defined and the finding the "linear" region becomes subjective. Fitting the full data to the form given in Eq.(11) makes it possible to extract $\mathcal{X}^{\infty}$ even when the system size is not much bigger than the correlation length. We illustrate this below using previously published data of Rovere, Nielaba and Binder [24] obtained from simulations of the lattice gas model using spin exchange dynamics (canonical ensemble).

In Fig. (2) we plot $\mathcal{X}^{L_{b}}$ as a function of $L / L_{b}=1 / x$. This choice of the axes is identical to the one used in the original work [24] and is equivalent. The points are the data of Ref. [24] at various (dimensionless) temperatures $T / J \geq T_{c} / J=2.2699$. The curves through the data are fits to Eq.(11). The extracted compressibility $\mathcal{X}^{\infty}$ are plotted in Fig. (3) and compared with the previous estimates [24 based on linear extrapolation and the theoretical curve [25]. Though, for large $T$ where the correlation length $\xi$ remains small one gets identical results, $\mathcal{X}^{\infty}$ extracted from the fits to the full curve continues to be closer to the theoretical (exact) estimate as $T \rightarrow T_{c}$ (and consequently $\xi \rightarrow \infty$ ). Simultaneously, we also obtain from Eq.(11) an estimate for the correlation length $\xi$ which is shown in Fig. (4). The agreement 
with theory [26] is not as good. This is only to be expected since the correlation length is a more sensitive quantity than the susceptibility. Also, the fitting procedure cannot overcome errors built into the data because of critical slowing down near $T_{c}$ which is known to lead to a systematic underestimation due to the use of a biased estimator [31].

Further, close to $T_{c}$ the free energy functional (Eq. (ID) ceases to be valid, the correlation function $G_{\phi \phi}(r) \sim|r|^{-(d-2+\eta)}$ and this affects our estimate for the correlation length. This is particularly important in two dimensions [26] where, in the Ising lattice gas, the exponent $\eta=1 / 4$ indicates a rather large deviation of the correlation function from the Ornstein Zernike behavior, Eq. (2), while in $d=3, \eta \sim 0.03$ and hence Eqs.(55) and (6) should be more accurate.

Far away from a critical point, on the other hand, none of these criticisms apply and accurate estimates for the susceptibility can be obtained even from relatively small systems even in $d=2$ using the ideas described here.

\section{STRAIN FLUCTUATIONS AND ELASTIC CONSTANTS}

\section{A. Theory}

Imagine a system in the constant NVT (canonical) ensemble at a fixed density $\rho=$ $N / V$ evolving in time $t$. For any "snapshot" of this system taken from this ensemble, the

local instantaneous displacement field $\mathbf{u}_{\mathbf{R}}(t)$ defined over the set of lattice vectors $\{\mathbf{R}\}$ of a reference lattice (at the same density $\rho$ ) is

$$
\mathbf{u}_{\mathbf{R}}(t)=\mathbf{R}(t)-\mathbf{R}
$$

where $\mathbf{R}(t)$ is the instantaneous position of the particle tagged by the reference lattice point R. In this paper we concentrate only on perfect crystalline lattices; if topological defects such as dislocations are present the analysis below needs to be modified. The instantaneous Lagrangian strain tensor $\epsilon_{i j}$ defined at $\mathbf{R}$ is then given by [8, [], 


$$
\epsilon_{i j}=\frac{1}{2}\left(\frac{\partial u_{i}}{\partial R_{j}}+\frac{\partial u_{j}}{\partial R_{i}}+\frac{\partial u_{i}}{\partial R_{k}} \frac{\partial u_{k}}{\partial R_{j}}\right)
$$

The strains considered here are always small and so we, hereafter, neglect the non -linear terms in the definition given above for simplicity. The derivatives are required at the reference lattice points $\mathbf{R}$ and can be calculated by any suitable finite difference scheme once $\mathbf{u}_{\mathbf{R}}(t)$ is known. We are now in a position to define coarse grained variables $\epsilon_{i j}^{L_{b}}$ which are simply averages of the strain over a sub-block of size $L_{b}$. The fluctuation of this variable then defines the size dependent compliance matrix $S_{i j k l}=<\epsilon_{i j} \epsilon_{k l}>$. Before proceeding further, we introduce a compact Voigt notation (which replaces a pair of indices $i j$ with one $\alpha$ ) appropriate for two dimensional strains - the only case considered in this paper. Using $1 \equiv x$ and $2 \equiv y$ we have,

$$
\begin{aligned}
& i j=112212 \\
& \alpha=1 \quad 2 \quad 3
\end{aligned}
$$

The only nonzero components of the compliance matrix are

$$
\begin{aligned}
& S_{11}=<\epsilon_{x x} \epsilon_{x x}>=S_{22} \\
& S_{12}=<\epsilon_{x x} \epsilon_{y y}>=S_{21} \\
& S_{33}=4<\epsilon_{x y} \epsilon_{x y}>
\end{aligned}
$$

It is also useful to define the following linear combinations

$$
\begin{aligned}
& S_{++}=<\epsilon_{+} \epsilon_{+}>=2\left(S_{11}+S_{12}\right) \\
& S_{--}=<\epsilon_{-} \epsilon_{-}>=2\left(S_{11}-S_{12}\right)
\end{aligned}
$$

Where $\epsilon_{+}=\epsilon_{x x}+\epsilon_{y y}$ and $\epsilon_{-}=\epsilon_{x x}-\epsilon_{y y}$. Once the block averaged strains $\epsilon_{i j}^{L_{b}}$ are obtained, it is straight -forward to calculate these fluctuations (for each value of $L_{b}$ ).

Since we are interested in the elastic properties of the system far away from any phase transition, a quadratic functional for the Helmholtz free energy $F$ suffices. We therefore 
use the following Landau functional appropriate for a two dimensional solid involving coarse grained strains to quadratic order in strains and its derivatives.

$$
\begin{aligned}
F= & \int d^{d} r\left\{c_{1} \epsilon_{+}^{2}+c_{2} \epsilon_{-}^{2}+c_{3} \epsilon_{3}^{2}+d_{1}\left(\nabla \epsilon_{+}(\mathbf{r})\right)^{2}\right. \\
& \left.+d_{2}\left(\nabla \epsilon_{-}(\mathbf{r})\right)^{2}+d_{3}\left(\nabla \epsilon_{3}(\mathbf{r})\right)^{2}\right\}
\end{aligned}
$$

The coefficients $c_{i}$ and $d_{i}$ are, of course, not all independent because of further symmetries [8.77] which are present for the two dimensional triangular solid though this does not influence the rest of our analysis. Note that, Eq. (18) is simply a sum of three independent functionals in $\epsilon_{+}, \epsilon_{-}$and $\epsilon_{3}$ each of the form given in Eq.(1). Thus the analysis described in detail in section II carries over almost unchanged. There is one small difference, however. Even in the canonical ensemble with fixed box dimensions, the microscopic strain fluctuations over the whole box are not zero but remains a small number of the order of $(a / L)^{2}$ where $a$ is the lattice parameter so that,

$$
\int^{L} d^{d} r<\epsilon_{\alpha}(\mathbf{r}) \epsilon_{\beta}(0)>=C_{\alpha \beta}\left(\frac{a}{L}\right)^{2}
$$

Incorporating this modification into Eq.(11) we get,

$$
\begin{aligned}
S_{\gamma \gamma}^{L_{b}}= & S_{\gamma \gamma}^{\infty}\left[\Psi(x L / \xi)-\left(\Psi(L / \xi)-C\left(\frac{a}{L}\right)^{2}\right) x^{2}\right] \\
& +\mathcal{O}\left(x^{4}\right) .
\end{aligned}
$$

Where the index $\gamma$ takes the values,+- or 3 and $x=L_{b} / L$ and we have suppressed subscripts on $\xi$ and $C$ for clarity. The above equation Eq.(20) can now be used to obtain the system size independent quantities $S_{\alpha \beta}^{\infty}, \xi$ and $C$.

Once the finite size scaled compliances are obtained the elastic constants viz. the Bulk modulus $B=\rho \partial P / \partial \rho$ and the shear modulus $\mu$ are obtained simply using the formulae 20

$$
\begin{aligned}
& \beta B=\frac{1}{2 S_{++}} \\
& \beta \mu=\frac{1}{2 S_{--}}-\beta P \\
& \beta \mu=\frac{1}{2 S_{33}}-\beta P
\end{aligned}
$$


where we assume that the system is under an uniform hydrostatic pressure $P$. The two expressions for $\mu$ should give identical results and constitutes an excellent internal check for numerical accuracy.

This completes the description of our technique for obtaining elastic constants. It is obvious that throughout the derivation we do not ever refer to the interparticle interactions. The only place where this information is required is in the calculation of the pressure $P$ of the system - a quantity routinely calculated in simulations. This, as we show later, is not a limitation even for systems with hard core potentials, where the calculation of pressure is more involved [17]. We now describe our results for two model, two dimensional, solids. The elastic constants in each case are obtained for a high density perfect triangular solid. The generalization of this method for higher dimensions, more complicated (less symmetrical) crystal types and even for amorphous materials is straightforward.

\section{B. Hard disks}

Hard disks interact with the extremely short ranged potential,

$$
\begin{aligned}
\beta V(r) & =0 \text { for } r>\sigma \\
& =\infty \quad r \leq \sigma
\end{aligned}
$$

where $\sigma$ the hard sphere diameter can be used as the unit of length and there is no energy scale. This makes the hard disk system particularly easy to simulate and is a popular testing ground for theories and techniques [6]. On the other hand, a calculation of elastic constants in this system is difficult since a harmonic description for such a solid does not exist at zero temperature. The energy vanishes and the free energy is wholly entropic in origin. For this reason most computational methods for obtaining elastic constants which work for smooth potentials have to be either discarded or modified in order to study elastic

properties of hard systems [16 18]. The only previous study of the hard disk elastic moduli is by Wojciechowski and Brańkai [18]. These authors carried out a Monte Carlo simulation 
of 56 hard disks in a box of variable shape in the constant stress ensemble [6]. The elastic constants were obtained from the fluctuations in the shape of the entire box. Results were checked for finite size effects by repeating a few test runs for 24,30 and 90 particles.

In our method the results derived in the previous sections carry over without any change to systems of particles interacting with hard potentials. Further we automatically calculate finite size scaled quantities. We present results for elastic constants of the hard disk system in two dimensions for a range of densities $0.95<\rho^{*}<1.1$ from Monte Carlo (MC) simulations with systems with sizes varying from 168 to 12480 particles. The simulations have been set up for a perfect triangular lattice in a slightly rectangular simulation box with periodic boundary conditions. The number of cells along each side of the box is adjusted to make the simulation box as close to a square as possible. In the hard disk system one can considerably accelerate the MC dynamics using special updating schemes [6]. We use a square grid as an overlay on the simulation box, and choose the grid size to be small enough to accommodate only one disk. An occupancy list of the grid positions is computed and continuously updated. For an attempted move of a hard disk from one grid point to another, we first check if the new point is unoccupied and then check for overlap only with the particles occupying the neighboring grid points. At the density $\rho \sigma^{2}=\rho^{*}=1$ a standard simulation had the length of $3 \times 10^{6} \mathrm{MC}$ steps, every 10-th MC step has been taken for averaging observables, in particular the block analysis was done for 100 random placements of the block.

In Fig. (5) we show the strain- strain fluctuations as a function of the relative sub-block size computed in a system with $N=3120$ hard disks at a density of $\rho^{*}=1.0$. The data are fitted to Eq.(20) where we keep terms up to order $\left(L_{b} / L\right)^{4}$. The fits to Eq.(20) are excellent and the infinite system compliances $S_{\alpha \beta}^{\infty}$ can be determined immediately. To determine the sensitivity of our results to the total system size we have also simulated systems with $N=12480,780$ and an extremely small system consisting of $N=168$ particles. Using the "infinite" system values determined from the $N=3120$ particle system it is, in principle, possible to predict the behaviour of the strain fluctuations $S_{33}\left(L_{b}\right)$ for the other systems. In Fig. (6) we have plotted $S_{33}\left(L_{b}\right)$ for $12480,3120,780$ and 168 particles. The points are the 
simulation data while the curves are fits to Eq. (20) where the value of $S_{33}^{\infty}$ was constrained to be fixed at that obtained from the data for 3120 particles. The results are seen to be almost insensitive to the total system size as expected.

Once the compliances $S_{\gamma \gamma}$ are obtained the elastic constants, in units of $k_{B} T / \sigma^{2}$, can be derived immediately using Eq.(21-23). Our results for densities other than $\rho^{*}=1.0$ were obtained from Monte Carlo simulations for $N=3120$ hard disks. To obtain the pressure, which is required for evaluating the shear modulus $\mu$, we simply integrate our bulk modulus $B$ (independent of the pressure in two dimensions) starting from the rather high density $\rho^{*}=1.1$ where the free volume [32] expression for the pressure $\beta P \sigma^{2}=P^{*}=2 \rho /(2 / \sqrt{3} \rho-1)$ is accurate. Our results [33] for the equation of state for the hard disk system is shown in Fig. (7) and those for the elastic constants are shown in Fig. (8). The two expressions for the shear modulus in Eq.22,23) give almost identical results and this gives us confidence about the internal consistency of our method. We have also compared our results to those of Wojciechowski and Brańka [18]. We find that while their values of the pressure and bulk modulus are in good agreement with ours (and with free volume theory) they grossly overestimate the shear modulus. This is probably due to the extreme small size of their systems and/or insufficient averaging. Our results for the sub-block analysis shows that finite size effects are non -trivial for elastic strain fluctuations and they cannot be evaluated by varying the total size of the system from 24 to 90, an interval which is less than half of a decade [34. One immediate consequence of our results is that the Cauchy relation 18, 35 $\mu=B / 2-P^{*}$ is seen to be valid upto $\pm 15 \%$ over the entire density range we studied (see Fig. (9) ) though there is a systematic deviation which changes sign going from negative for small densities to positive as the density is increased. This is in agreement with the usual situation in a variety of real systems 35 with central potentials and highly symmetric lattices and in disagreement with Ref. [18]. We have also compared our estimates for the elastic constants with the density functional theory (DFT) of Rhysov and Tareyeva [36]. We find that both the bulk and the shear moduli are grossly overestimated - sometimes by as much as $100 \%$. 


\section{Soft disks}

The system of particles in two dimensions interacting by a purely repulsive inverse $12^{\text {th }}$ power pair potential $v\left(r_{i j}\right)$ of the form given by,

$$
v(r)=e\left(\frac{\sigma}{r}\right)^{12}
$$

has been studied [37,38] quite extensively. This system has the advantage of being realistic without being too complicated, since the form of the potential ensures that the entire equation of state can be determined from that of a single isotherm [37]. The quantities $e$ and $\sigma$ sets the scales for energy and distance respectively and can be both set equal to unity. Both the zero and the finite temperature elastic constants of this system has been calculated over a large range of densities [37]. We have used this system to test the applicablity of our method to molecular dynamics simulations. Our results here are not as extensive as in the hard disk case and we obtain elastic constants only for a single state point.

We simulate this system with a simple leap -frog molecular dynamics code incorporating a Nosé Hoover thermostat [6] in order to generate configurations in the canonical NVT ensemble. The temperature $T^{*}=k_{B} T / e$ is fixed at 1 and the density $\rho^{*}=\rho \sigma^{2}$ at $1.05-$ a state sufficiently far from melting. The number of particles were chosen to be $N=780$ which is same as that used in Ref. [37] and corresponds to $26 \times 30$ unit cells of a triangular lattice within a nearly square box. Starting from the perfect lattice initial configuration, the system was equilibrated for more than $10^{6}$ molecular dynamics time steps $\Delta t=0.002$ ( in units of $\sqrt{m \sigma^{2} / e}$ where $m$ is the mass of the particles). Subsequently, averages of thermodynamic quantities were calculated over $\approx 10^{4}$ uncorrelated configurations. Our results for the elastic compliances $S_{\gamma \gamma}$ are similar to that in the hard disk case and are shown in Fig. (10).

The final estimates for the bulk modulus $B=77.96$ and shear modulus $\mu=23.34$ (in units of $\left.k_{B} T / \sigma^{2}\right)$ compare well with those of Ref. [37 (viz. $B=79.71$ and $\mu=24.96$ ). Errors in our estimate for the bulk modulus arise from statistical error in the acquired data and from the fits this is around 3\%. The shear modulus being a more sensitive quantity to 
compute is less accurate and the two expressions for $\mu$ in Eq. (22,23) now differ by $10-15 \%$ (the number quoted above is the average value). This maybe due to the smaller size of our system compared to the hard disk case, and because subsequent configurations in molecular dynamics simulations are more correlated than in Monte Carlo.

\section{CONCLUSION}

In summary, we show in this paper that a systematic coarse graining analysis of strain fluctuations yields elastic constants of solids from computer simulations to high accuracy. Our method incorporates finite size scaling and produces elastic constants in the thermodynamic limit. The procedure is simple to implement and is general enough to be carried out for any system without modification. Before we end this paper, the following comments are, perhaps, in order.

The coarse graining variable:- Firstly, we introduced this work as a "test" case of a general coarse graining procedure which may be used to study the connection between microscopic computer simulations and long wavelength physics contained in continuum field theory approaches for phase transitions in solids. In this regard we would like to point out that we found, as usual, the choice of the coarse graining variable to be important. Our results show that microscopic strains calculated by taking finite differences of the displacement field constitute the correct variable to coarse grain over. One could alternatively have averaged the displacement field $\mathbf{u}(\mathbf{r})$ and calculated the strains from the coarse grained $\mathbf{u}$. This procedure happens to produce wrong results giving us elastic constants which are orders of magnitude too large.

The strain correlation length:- One of the new results from our calculations is the correlation length $\xi_{\alpha \beta}$ of strain fluctuations. This is found to be small - $2-3$ lattice spacings - for all components of the strain-strain correlation functions and for both the systems. We have checked this independently by explicitly measuring the correlation function $G_{33}(\|\mathbf{R}\|)$

defined for all the lattice vectors $\mathbf{R}$ of the two dimensional triangular reference lattice in the 
soft sphere system. Though, the simple Ornstein Zernicke form is inadequate to describe detailed features of the correlation function and actual values for the correlation length is hard to estimate, preliminary results from our simulations do support the above contention. The correlation of the local density $\rho(\mathbf{r})$ or its phase - the displacement field $\mathbf{u}(\mathbf{R})$ is, of course, long ranged, decaying algebraically as it should in the solid state.

Renormalization by defects:- Our results for the elastic constants are obtained for high density perfect solids. In general a solid contains point (vacancies and interstitials) and line (dislocations) defects. For example, in the hard disk case dislocations start appearing in our systems below a density of $\rho^{*}=0.95$ a range we have not explored in this paper. In principle, there is no reason why our method cannot be adapted for systems containing defects though this involves considerable computational complexity. There are basically two problems which arise when one wishes to calculate elastic constants in the presence of defects. Firstly, we have to ensure that the density of each type of defect on the average attain their equilibrium value. This is nontrivial because nucleation barriers for defects densities are usually high which means large system sizes and long simulation times are required. Defect mobilities are sluggish in a solid but to ensure that they are fully equilibrated one has to wait long enough to allow a typical dislocation to travel a distance equal to the system size [39]. Secondly, once we are sure that our configurations contain, on the average, the required defect densities, we have to evaluate the strain field in presence of these defects. This is, by far, the easier part. Defects can be either isolated point defects which arise in grand canonical simulations or vacancy -interstitial pairs which nucleate pairs of dislocations of opposite sign. The concentration of point defects in solids is vanishingly small $\sim 10^{-2}-10^{-4}$ atomic percent and they are not expected to change the elastic constants substantially. In any case they can be easily taken into account by redefining the reference lattice points. Topological defects introduce, in addition, a singular part in the displacement field so that the strain field cannot now be evaluated simply by taking numerical derivatives. However, the singular contribution for each dislocation is known analytically - so that for a given configuration one can locate topological defects of each type and treat the smooth and the 
singular parts separately. Lastly, we would like to point out that the above two problems viz. (1) obtaining an equilibrium defect concentration and (2) evaluating the finite size scaling of singular strain fields of configurations containing defects are present in all techniques for calculating elastic constants although they seem not to have recieved so far the attention they deserve.

The renormalization of elastic constants by dislocations can also be obtained approximately by using standard recursion relations [12 once the core energy of a dislocation is known (for instance from a separate simulation) and the present (manifestly "bare") values of the elastic constants are used as inputs. Such a calculation has applications in a study of two dimensional melting [11,12]. We are, at present, carrying out detailed calculations of the elastic constants, equation of state and dislocation core energies of the hard disk and inverse power triangular solids to investigate their melting behaviour. Further, near to the melting transition additional finite size effects would be manifest (due to diverging correlation lengths) and they have to be taken into account separately which would necessarily involve simulations of a much larger scale than employed here. For example, it is known that near the liquid to hexatic transition $\left(\rho^{*}=0.89\right) N=256^{2}$ hard disks are required 40 in order to reach the thermodynamic limit.

Evaluation of local stresses:- Lastly, we would like to point out that our procedure can also be used in an "inverse" mode where knowing the elastic constants for a system, strain fluctuations can be used to calculate local stresses. This is especially useful in experimental studies 41,42 of melting behaviour of colloidal particles where high quality digitized particle images are available. Efforts in this direction are in progress.

\section{ACKNOWLEDGMENTS}

We thank Hartmut Löwen and M. Müser for discussions. One of us (S.S.) thanks the Alexander von Humboldt Foundation for a fellowship. Partial support by the SFB 513 is gratefully acknowledged. 


\section{REFERENCES}

[1] C. Godrèche ed. Solids far from equilibrium, (Cambridge, Cambridge University Press, 1992).

[2] Z. Nishiyama, Martensitic Transformations, New York, Academic (1979); A. Roitburd, in Solid state physics, eds. H. Ehrenreich, F. Seitz and D. Turnbull (Academic Press, New York, 1958); G. R. Barsh and J. A. Krumhansl, Phys. Rev. Lett. 37, 9328 (1974); M. Rao and S. Sengupta, Phys. Rev. Lett. 78, 2168 (1997).

[3] A. Bishop and T. Schneider eds. Solitons in Condensed Matter Physics, (Berlin, Springer, 1978); D. Nelson and S. Weinberg eds., Statistical Mechanics of Membranes and Surfaces (Singapore, World Scientific 1989)

[4] B. N. J. Perron, E. Tosatti eds. Physics of Sliding Friction, NATO ASI Series, (Kluwer, Derchrecht, 1996); G. He, M. H. Müser and M. O. Robbins, Science (in press).

[5] B. Lawn, Fracture in Brittle Solids, (Cambridge University Press, Cambridge, 1975), 2nd Ed.; L. B. Freund, Dynamic Fracture Mechanics, (Cambridge University Press, New York, 1990); D. Holland and M. P. Marder, Phys. Rev. Lett. 81, 4029 (1998).

[6] M. P. Allen and D. J. Tildesley Computer Simulation of Liquids, (Clarendon Press, Oxford 1990); D. Frenkel and B. Smit, Understanding Molecular Simulations (Academic Press, San Diego, 1996).

[7] L. D. Landau and E. M. Lifshitz, Theory of Elasticity 3rd Edition, (Pergamon, Oxford, 1986).

[8] P. M. Chaikin and T. C. Lubensky, Principles of condensed matter physics, (Cambridge University Press, Cambridge, 1995).

[9] R.M. Lynden-Bell and K.H. Michel, Rev. Mod. Phys. 66, 721 (1994); B. De Raedt, K. Binder and K.H. Michel, J. Chem. Phys. 75, 2977 (1981). 
[10] A.D. Bruce and R.A. Cowley, Structural Phase Transitions, London, Taylor and Francis (1981); R.A. Cowley, Phys. Rev. B 13, 4877 (1976); R. Folk, H. Iro and F. Schwabl, Z. Phys. B 25, 69 (1976);K. Binder in Phase Transformations in Materials (P. Haasen ed.) p 143 VCH, Weinheim (1991); L. Delaye, ibid.

[11] B.I. Halperin and D.R. Nelson, Phys. Rev. Lett. 41, 121 (1978); A.P. Young, Phys. Rev. B 19, 1855 (1979); K.J. Strandburg, Rev. Mod. Phys. 60, 161 (1988); H. Kleinert, Gauge Fields in Condensed Matter, Singapore, World Scientific (1989).

[12] D. R. Nelson and B. I. Halperin, Phys. Rev. B , 19, 2457 (1979).

[13] S.T. Chui, Phys. Rev. Lett. 48, 933 (1982); Phys. Rev. B 28, 178 (1983).

[14] M. Parrinello and A. Rahman, J. Chem Phys. 76, 2662 (1982); A.A. Gusev, M,M, Zehnder and U.W. Suter, Phys. Rev. B 54, 1, (1996).

[15] D. R. Squire, A. C. Holt and W. G. Hoover, Physica 42, 388 (1969).

[16] D. Frenkel and A. J. C. Ladd, Phys. Rev. Lett. 59, 1169 (1987).

[17] K. J. Runge and G. V. Chester, Phys. Rev. A 36, 4852 (1987).

[18] K. W. Wojciechowski and A. C. Brańka, Phys. Lett. 134A, 314 (1988).

[19] J.A. Barker and D. Henderson, Mol. Phys. 21, 187 (1971).

[20] D. C. Wallace, in Solid state physics, eds. H. Ehrenreich, F. Seitz and D. Turnbull (Academic Press, New York, 1958); J. H. Weiner, Statistical mechanics of elasticity (Wiley, New York, 1983).

[21] K. Binder ed. Application of the Monte Carlo Method in Statistical Physics (Springer, Berlin, 1998).

[22] K. Binder, Z. Phys. B 43, 119 (1981).

[23] M. Rovere, D. W. Heermann and K. Binder, Europhys. Lett. 6, 585 (1988); J. Phys. 
Cond. Mat. 2, 7009 (1990).

[24] M. Rovere, P. Nielaba and K. Binder, Z. Phys. B 90, (1993).

[25] C. Domb in Phase Transition and critical phenomena, Vol. 3 (Academic Press, London, 1974).

[26] M.E. Fisher, R.J. Burford, Phys. Rev. 156, 583 (1967).

[27] F. L. Román, J. A. White and S. Velasco, Europhys. Lett. 42, 371, (1998); F. L. Román, J. A. White and S. Velasco, J. Chem. Phys. 107, 4635 (1997).

[28] S. Sengupta, D. Marx, P. Nielaba and K. Binder, Phys. Rev. E , 49, 1468 (1994).

[29] D. Marx, P. Nielaba and K. Binder, Phys. Rev. Lett. 67, 3124 (1991).

[30] J. L. Lebowitz, J. K. Percus and L. Verlet, Phys. Rev. 153, 250, (1967); K. W. Krathy, Phys. Rev. A 31, 945, (1985); J. J. Salacuse, A. R. Denton and P. A. Egelstaff, Phys. Rev. E 53, 2382 (1996).

[31] A. M. Ferrenberg, D. P. Landau and K. Binder, J. Stat. Phys. 63, 867, (1991).

[32] F. H. Stillinger and Z. W. Salzburg, J. Chem. Phys. 46, 3962, (1967).

[33] The results presented in this paper for the perfect triangular solid in the hard disk system was obtained by imposing a "single cell occupancy" constraint, rejecting all Monte Carlo moves which takes a particle out of its (hexagonal) Wigner -Seitz cell. This ensures that no defects are produced during the Monte Carlo runs though the effect of the constraint on elastic moduli for the densities considered is negligible. Below a density of $\rho^{*}=0.95$ this constraint becomes increasingly relevant.

[34] To eliminate the effects of finite size completely one has to use system sizes at least an order of magnitude larger than the correlation length. Our fits to Eq.(20) yeilds correlation lengths of about $2-3$ lattice spacings, which implies that we would need a system of at least $N=(2 \times 2 \times 10)^{2}=1600$ particles taking care of periodic boundary 
conditions. This is much larger than the sizes used by Wojciechowski and Branka.

[35] for general discussions on Cauchy relations see F. Seitz, Modern Theory of Solids (McGaraw-Hill, New York, 1940) and M. Born and K. Huang, Dynamical Theory of Crystal Lattices (Oxford University Press, Oxford, 1954); For experimental temperature dependent elastic constants of solid argon see, H. Meixner, P. Leiderer, P. Berberich and E. Lüscher, Phys. Lett. 40A, 257 (1972).

[36] V. N. Ryzhov and E. E. Tareyeva, Phys. Rev. B , 51, 8789 (1995).

[37] J. Q. Broughton, G. H. Gilmer and J. D. Weeks, Phys. Rev. B , 25, 4651 (1982).

[38] K. Bagchi, H. C. Andersen and W. Swope, Phys. Rev. E 53, 3794 (1996).

[39] A. Zippelius, B. I. Halperin and D. R. Nelson, Phys. Rev. B , 22, 2514, (1980).

[40] A. Jaster, eprint, cond-mat/9810274

[41] K. Zahn, R. Lenke and G. Maret, Phys. Rev. Lett. 82, 2721, (1999)

[42] Q. -H. Wei, C. Bechinger, D. Rudhardt and P. Leiderer, Phys. Rev. Lett. 81, 2606 (1998). 


\section{FIGURES}

FIG. 1. A plot of $\mathcal{X}^{L_{b}}$ as a function of the relative sub-block size $L_{b} / L$ as given by Eq.(11) for various values of the parameter $L / \xi(=1,5,10 \& 50)$.

FIG. 2. The susceptibility $\mathcal{X}^{L_{b}}(T)$ of a two dimensional Ising system in the constant magnetization ensemble as a function of the relative sub-block size $L / L_{b}$ for values of the temperature $T / J>T_{c} / J$. The symbols refer to simulation data of the critical lattice gas simulation of Ref. (23) and the lines are fits of Eq. (11) to these data.

FIG. 3. The estimates for $\chi_{\infty}(T)$ as obtained from the fits shown in Fig. 2. $(\diamond)$ compared with the estimates of Ref. $(23)(+)$. Note that the finite size scaling analysis described here yields estimates which are closer to the exact result ( curve ).

FIG. 4. The correlation length $\xi$ (in units of the lattice parameter $a$ ) as a function of the scaled temperature $T / J$ of the lattice gas model at the critical density. Symbols: our estimates from fits to the data of Ref. [24] and curve: theory Ref. [26].

FIG. 5. Strain strain fluctuations or elements of the compliance matrix $S_{\gamma \gamma}$ defined in Eq. (16) $(\gamma=+,-$ or 3$)$ as a function of relative sub-block size $L_{b} / L$, symbols: simulation data; curve:

fit to the scaling function Eq. (20). The results (symbols) shown are for a system of $N=3120$ hard disks at $\rho^{*}=1.0$. 
FIG. 6. The infinite system susceptibility $S_{33}^{\infty}$ obtained from the data for $N=3120$ particles is used to predict the finite size behaviour of $N=168,780$ and 12480 particles. For $N=168,780$ and 3120 the symbols are simulation data and the solid lines are the best fits to the form given in Eq. (20) where the parameter $S_{33}^{\infty}$ is kept fixed at the value obtained from fits to the data for 3120 particles. For $N=12480$ we have acquired data (symbols) only for $L / L_{b}=$ an integer (between 4 and 35) and the dotted line is a straight line with the slope given by the same $S_{33}^{\infty}$.

FIG. 7. The equation of state of the hard disk solid, pressure $P^{*}$ as a function of the density $\rho^{*}$. We compare our results $(\diamond)$ with those of Ref. [18] (+) and free volume theory (line).

FIG. 8. The bulk $(B)$ and shear $(\mu)$ moduli in units of $k_{B} T / \sigma^{2}$ for the hard disk solid. Our results for $B(\mu)$ are given by $\square(\diamond)$. The values for the corresponding quantities from Ref. [18] are given by + and $\times$. The line through the bulk modulus values is the analytical expression obtained from the free volume prediction for the pressure. The line through our shear modulus values is obtained from the free volume bulk modulus using the Cauchy relation $\mu=B / 2-P$. The error bars in our values for the shear modulus correspond to the two alternative formulae for evaluating $\mu$ as given in Eq. 22, 23) and represents our most conservative error estimates.

FIG. 9. The percentage deviation $\Delta_{c}$ of our shear modulus values $\mu$ from that obtained from our bulk moduli $B$ using the Cauchy relation $\mu=B / 2-P$ as a funtion of the density $\rho^{*}$. The error bars in this graph correspond to the two formulae for evaluating $\mu$ as given in Eq.(22,23) as in the previous figure.

FIG. 10. Same as in Fig. 5 but for $N=780$ soft disks interacting with the inverse $12^{\text {th }}$ power potential at $\rho^{*}=1.05$ and $T^{*}=1$. 


\section{FIGURES}

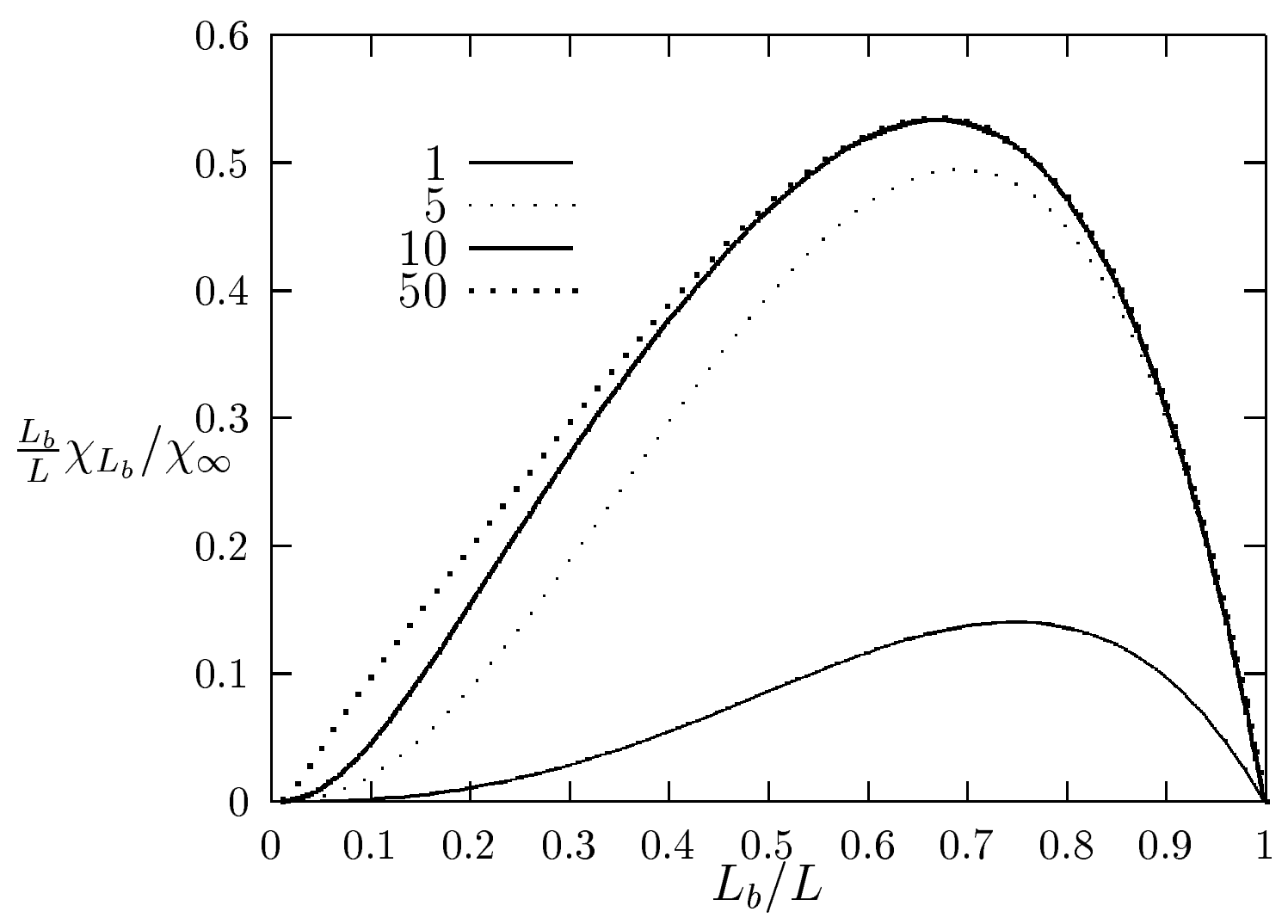

Figure 1:

(Sengupta, Nielaba, Rao, Binder; Phys. Rev. E) 
FIGURES

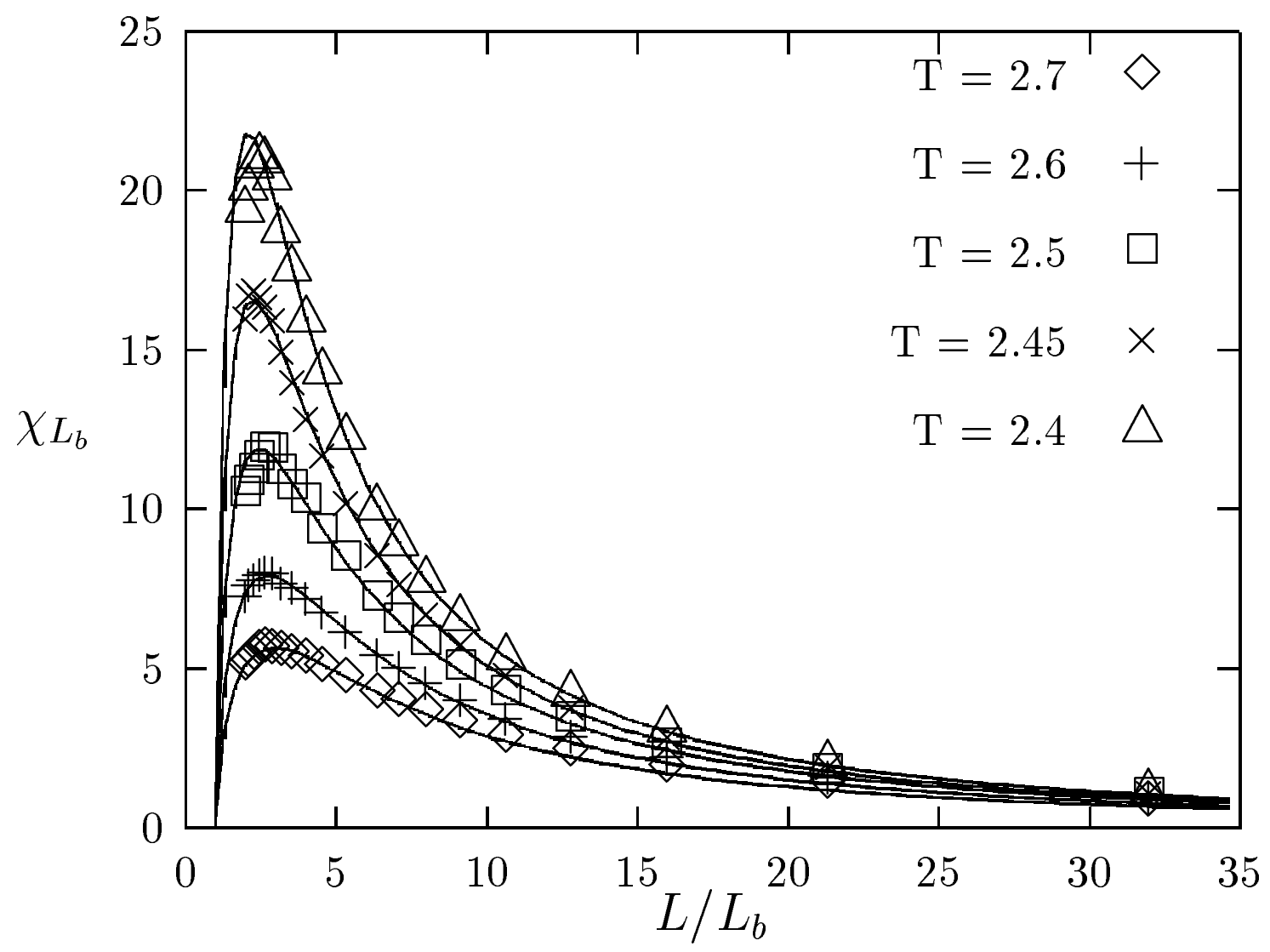

Figure 2:

(Sengupta, Nielaba, Rao, Binder; Phys. Rev. E) 
FIGURES

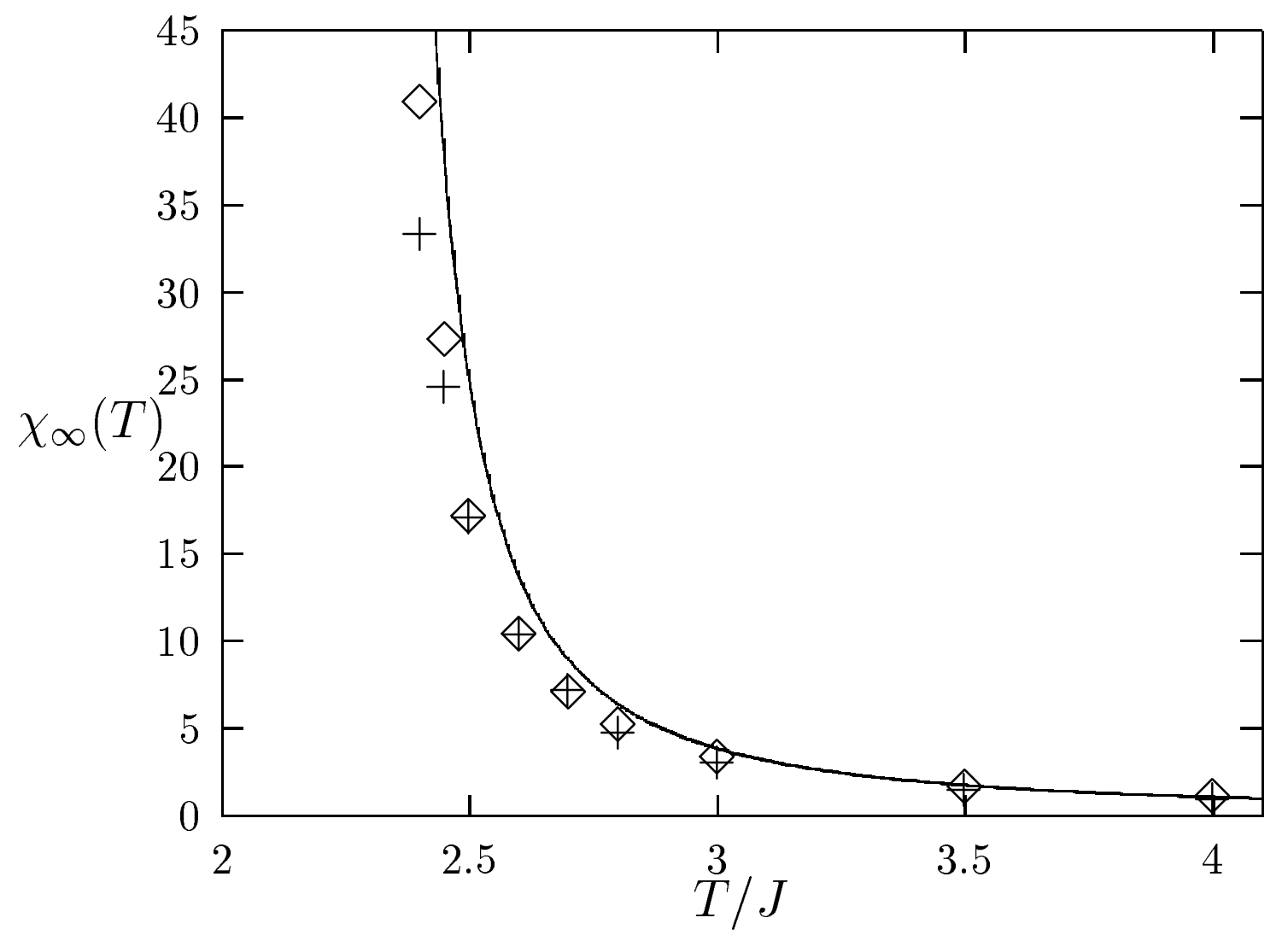

Figure 3:

(Sengupta, Nielaba, Rao, Binder; Phys. Rev. E) 
FIGURES

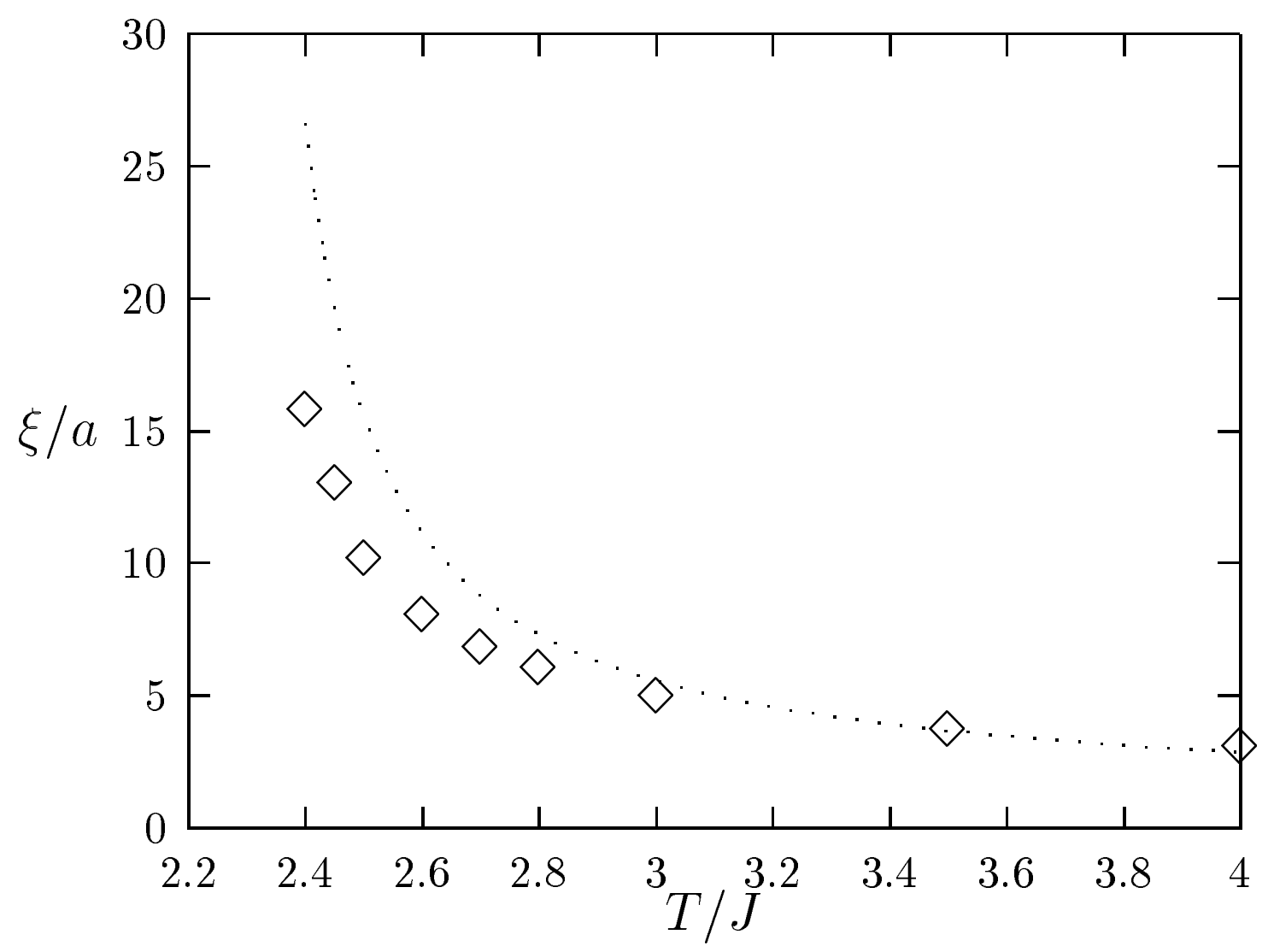

Figure 4:

(Sengupta, Nielaba, Rao, Binder; Phys. Rev. E) 


\section{FIGURES}

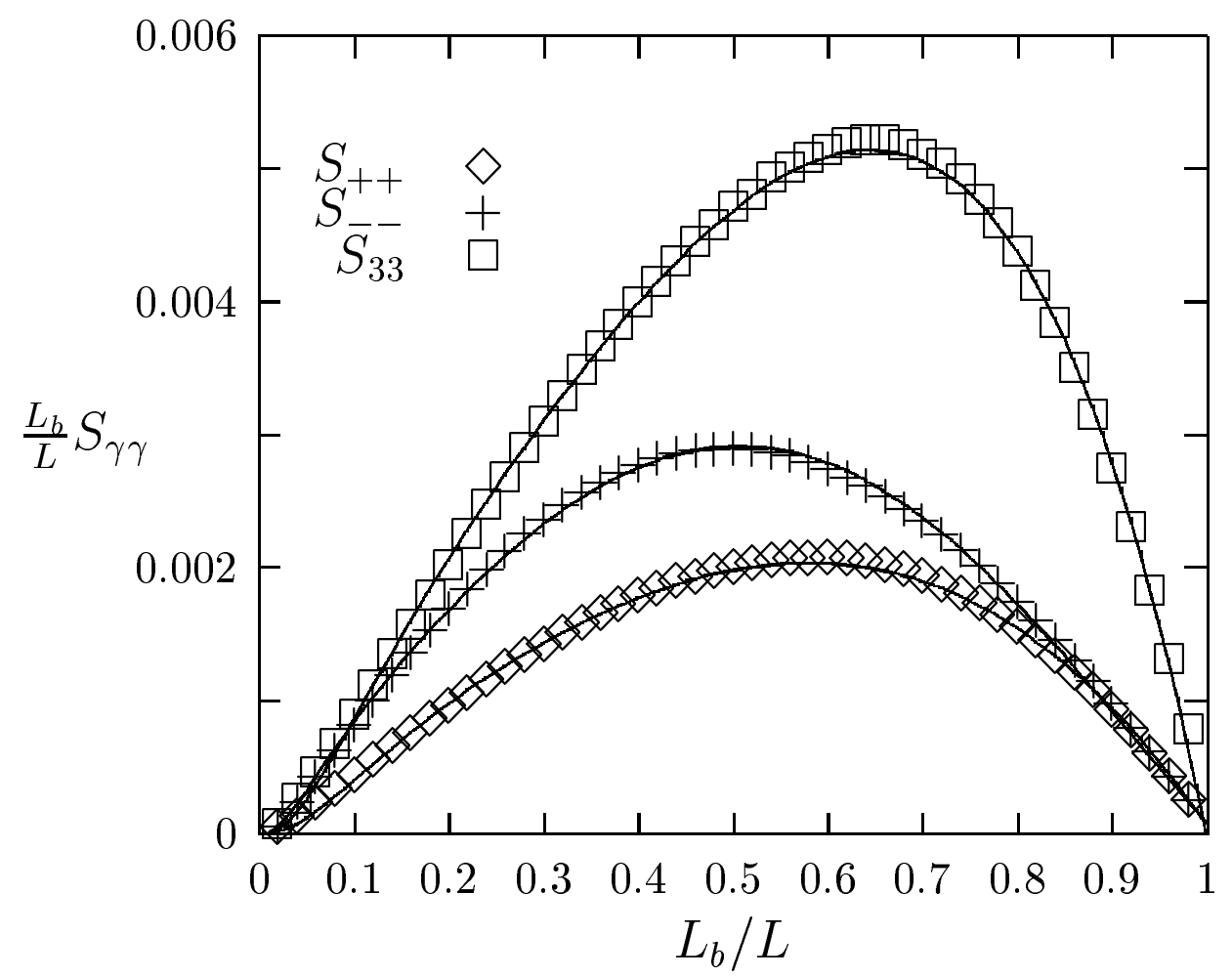

Figure 5:

(Sengupta, Nielaba, Rao, Binder; Phys. Rev. E) 


\section{FIGURES}

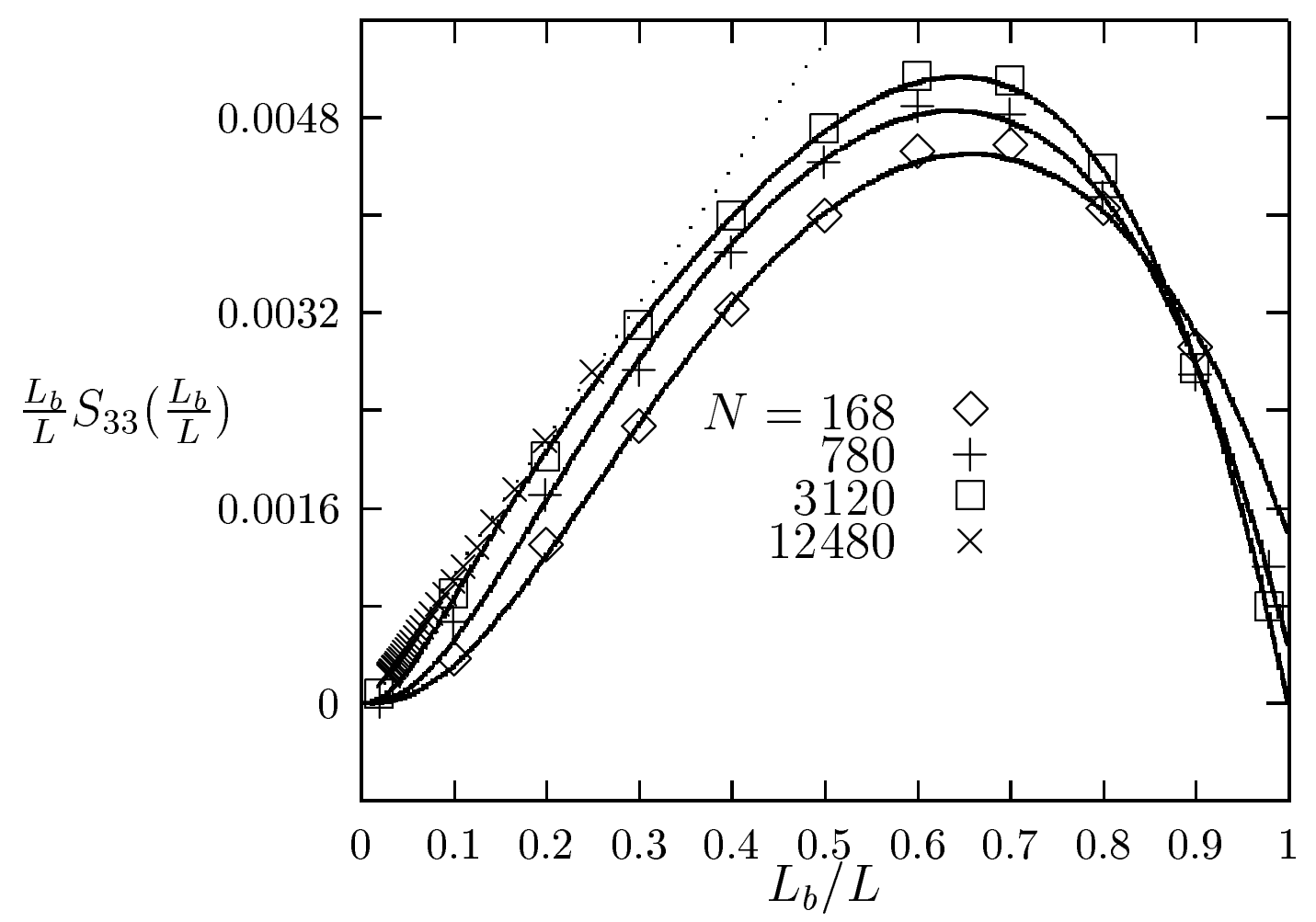

Figure 6:

(Sengupta, Nielaba, Rao, Binder; Phys. Rev. E) 


\section{FIGURES}

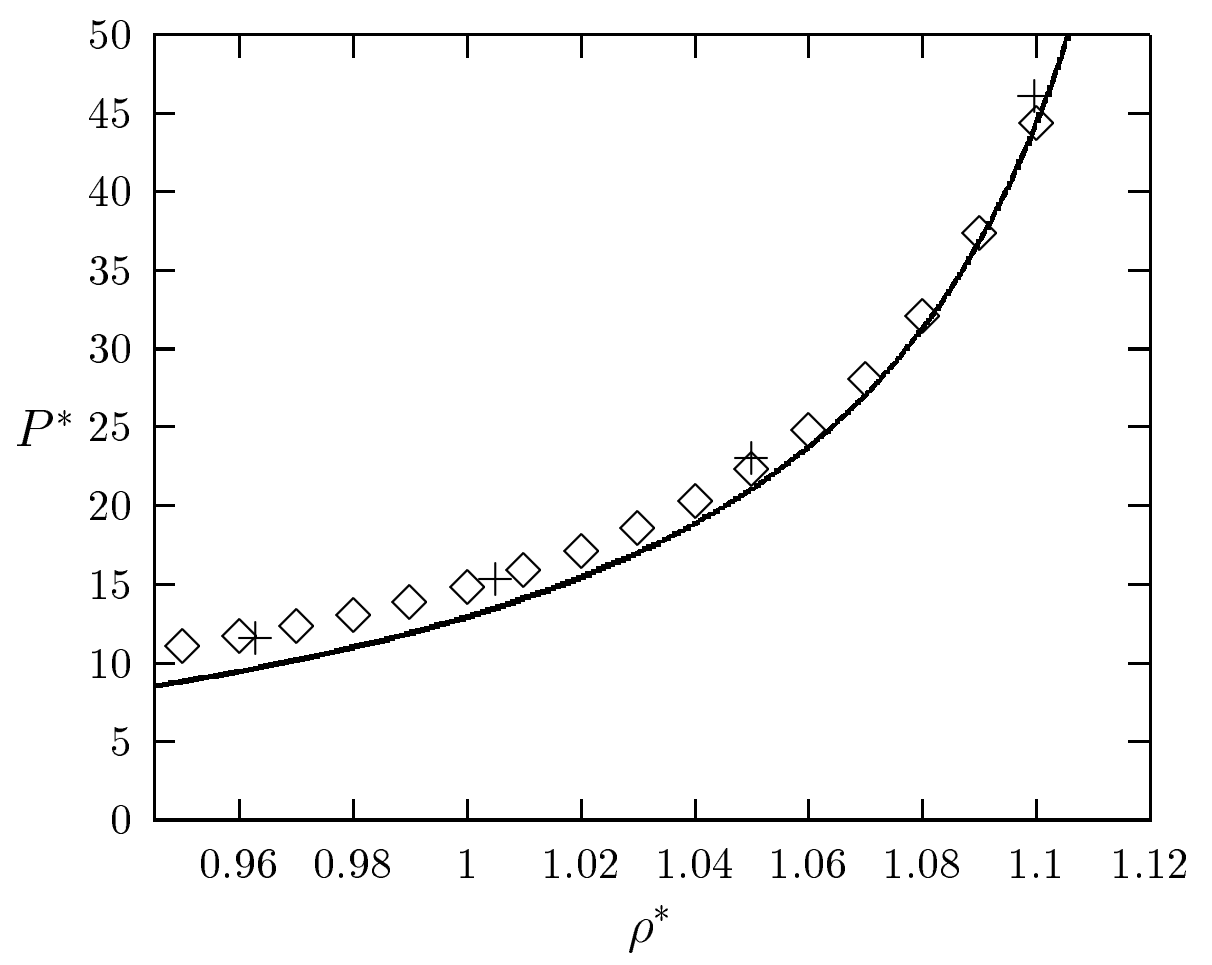

Figure 7:

(Sengupta, Nielaba, Rao, Binder; Phys. Rev. E) 


\section{FIGURES}

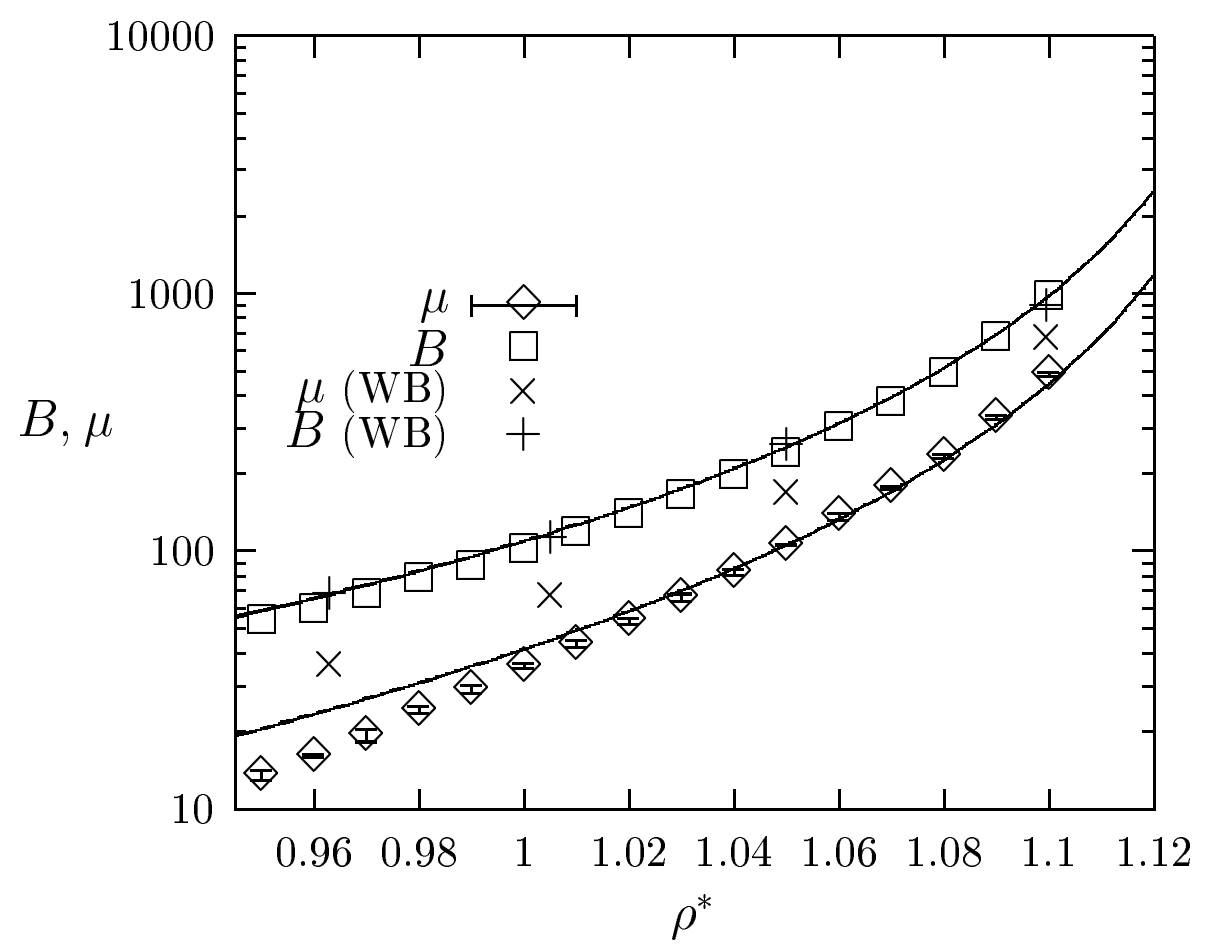

Figure 8:

(Sengupta, Nielaba, Rao, Binder; Phys. Rev. E) 


\section{FIGURES}

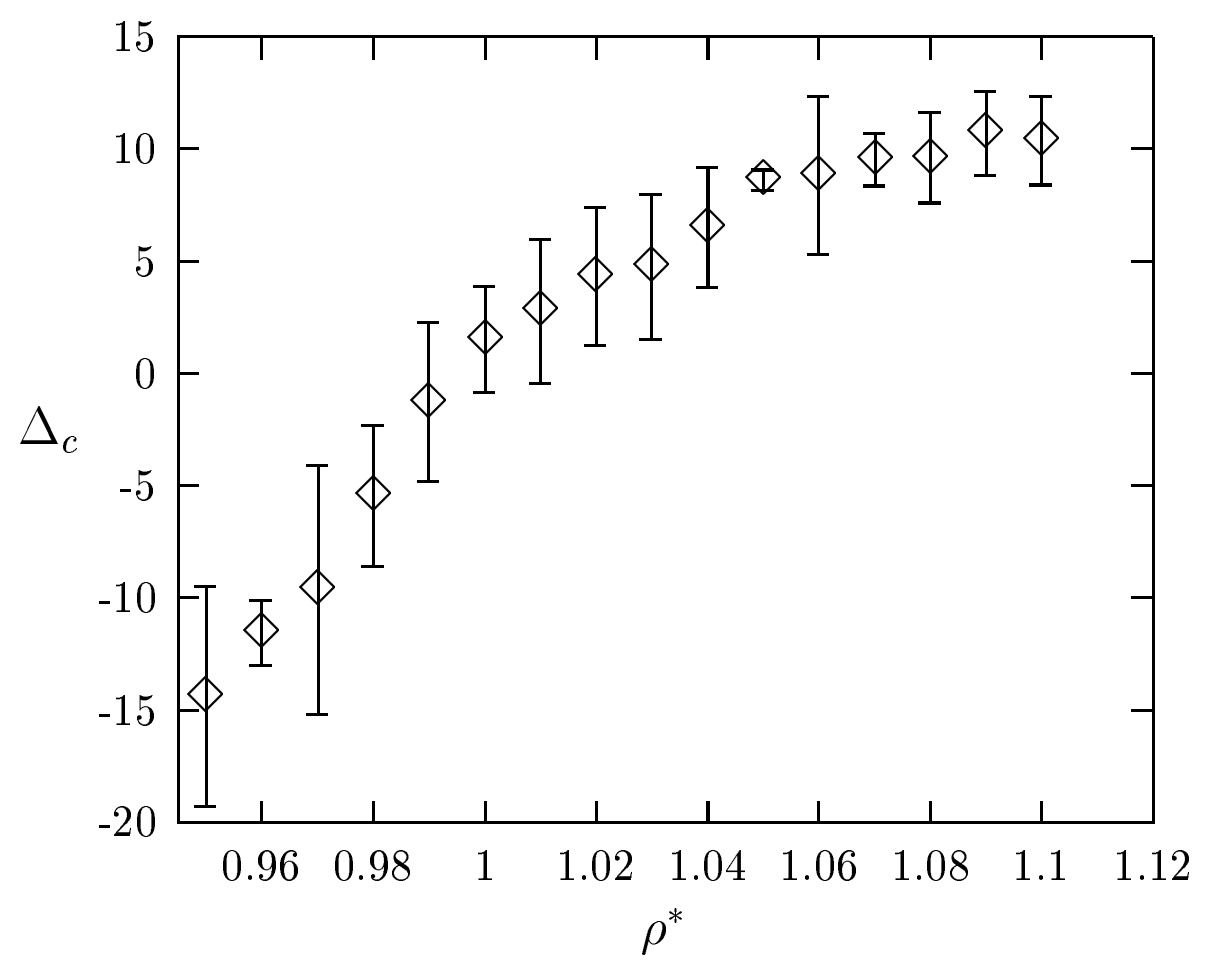

Figure 9:

(Sengupta, Nielaba, Rao, Binder; Phys. Rev. E) 


\section{FIGURES}

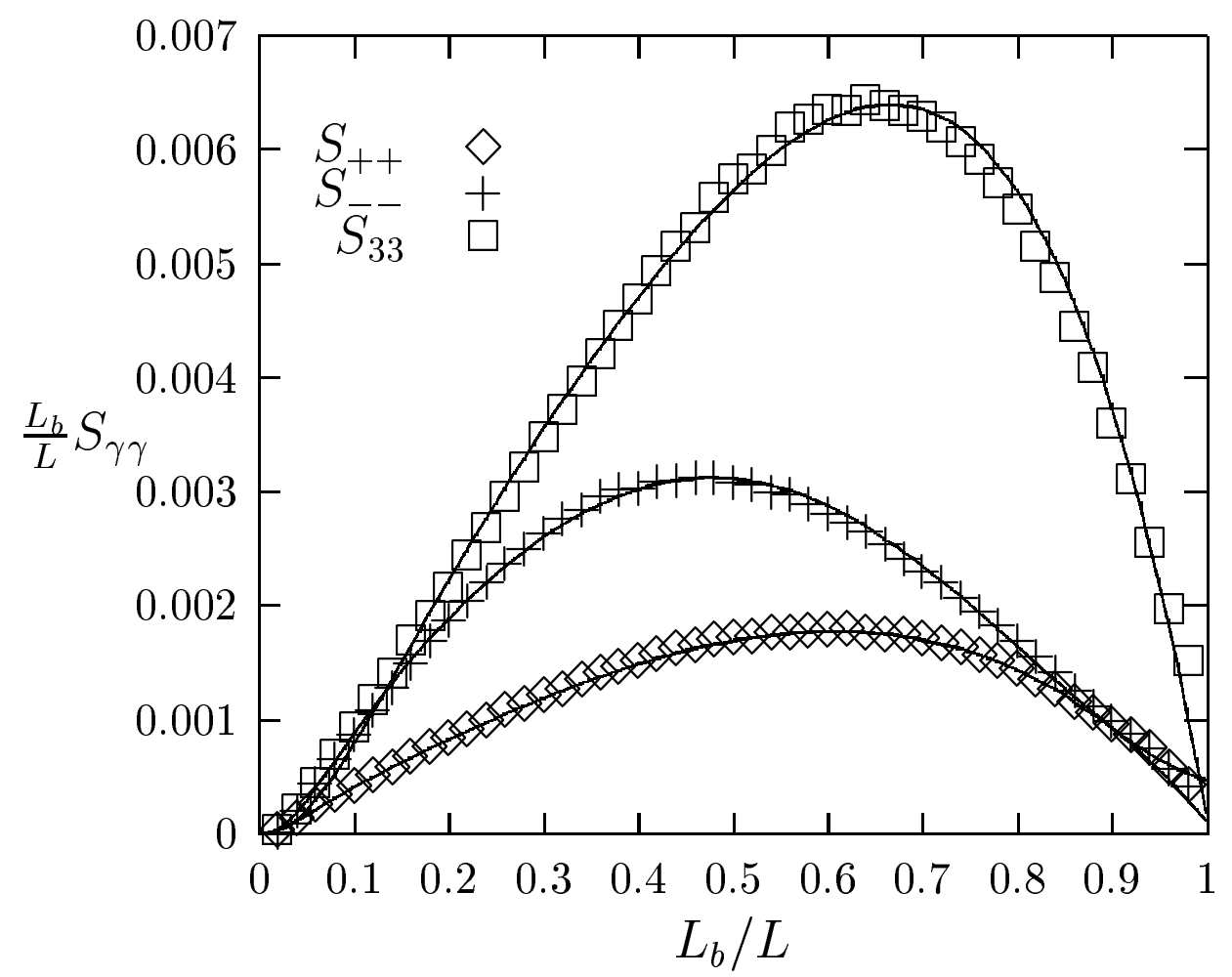

Figure 10:

(Sengupta, Nielaba, Rao, Binder; Phys. Rev. E) 